\title{
Correction to: Analysis of primary tumor metabolic volume during chemoradiotherapy in locally advanced non-small cell lung cancer
}

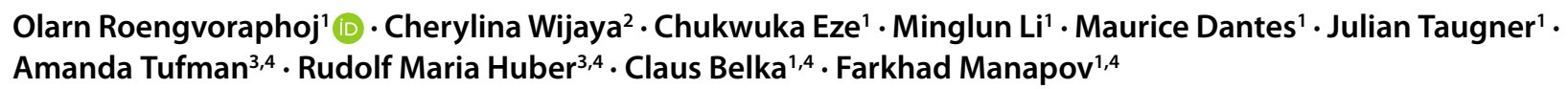

Published online: 14 March 2018

C Springer-Verlag GmbH Germany, part of Springer Nature 2018

\section{Correction to:}

\section{Strahlenther Onkol 2017}

https://doi.org/10.1007/s00066-017-1229-3

Unfortunately, the structure of Table 4 was incorrect. The corrected version of Table 4 can be found below. We apologize for any inconvenience caused.

The online version of the original article can be found under https://doi.org/10.1007/s00066-017-1229-3.

\section{Olarn Roengvoraphoj}

Olarn.Roengvoraphoj@med.uni-muenchen.de

1 Department of Radiation Oncology, University Hospital, LMU Munich, Munich, Germany

2 Department of Pulmonology, Asklepios Fachkliniken München-Gauting, Munich, Germany

3 Respiratory Medicine and Thoracic Oncology, Internal Medicine V, Ludwig-Maximilians-University of Munich and Thoracic Oncology Centre Munich, Munich, Germany

4 members of the German Centre for Lung Research (DZL CPC-M), Giessen, Germany 
Table 4 A summary of clinical trials showing correlation between PET/CT and overall survival in patients with NSCLC

\begin{tabular}{|c|c|c|c|c|c|c|}
\hline Study & $\begin{array}{l}\text { Patient } \\
\text { no. }\end{array}$ & $\begin{array}{l}\text { NSCLC } \\
\text { stage }\end{array}$ & $\begin{array}{l}\text { Pre-treatment } \\
\text { PET/CT }\end{array}$ & $\begin{array}{l}\text { Mid-treatment } \\
\text { PET/CT }\end{array}$ & $\begin{array}{l}\text { Post-treatment } \\
\text { PET/CT }\end{array}$ & Endpoint \\
\hline Machtay et al. (2013) [9] & 250 & $\begin{array}{l}\text { Inoperable } \\
\text { stage IIB/III }\end{array}$ & SUV peak & $\begin{array}{l}\text { Not per- } \\
\text { formed }\end{array}$ & SUV peak & $\begin{array}{l}\text { 2-Year OS post-treatment } \\
\text { SUVpeak }>5 \\
38 \% \text { vs. 53\% Observation arm } \\
(p=0.001)\end{array}$ \\
\hline Usmanij et al. (2013)[10] & 28 & $\begin{array}{l}\text { Inoperable } \\
\text { stage III }\end{array}$ & TLG & $\begin{array}{l}\text { Not per- } \\
\text { formed }\end{array}$ & TLG & $\begin{array}{l}\text { Delta TLG decrease of } 38 \% \\
\text { was associated with prolonged } \\
\text { PFS }\end{array}$ \\
\hline Van Elmpt et al. (2012) [11] & 34 & Stage II-IV & $\begin{array}{l}\text { SUVmean, } \\
\text { SUVmax }\end{array}$ & $\begin{array}{l}\text { SUVmean, } \\
\text { SUVmax }\end{array}$ & $\begin{array}{l}\text { Not per- } \\
\text { formed }\end{array}$ & $\begin{array}{l}\text { For patients with a decrease } \\
\text { in SUV }>15 \%, 2 \text {-year OS was } \\
92 \% \text {, compared with a median } \\
\text { OS of } 19 \text { months, and a } 33 \% \\
\text { 2-year OS, respectively, for } \\
\text { the patients with a decrease in } \\
\text { mean SUV of less than } 15 \%\end{array}$ \\
\hline Ohri et al. (2015) [24] & 214 & $\begin{array}{l}\text { Inoperable } \\
\text { stage IIB/III }\end{array}$ & MTV & $\begin{array}{l}\text { Not per- } \\
\text { formed }\end{array}$ & $\begin{array}{l}\text { Not per- } \\
\text { formed }\end{array}$ & $\begin{array}{l}\text { Median OS for patients } \\
\text { with MV }<95.4 \mathrm{~cm}^{3} 23.6 \text { vs. } \\
13.4 \text { months }(p<0.001)\end{array}$ \\
\hline $\begin{array}{l}\text { Markovina et al. } \\
(2015)[27]\end{array}$ & 176 & $\begin{array}{l}\text { Inoperable } \\
\text { stage IIB/III }\end{array}$ & $\begin{array}{l}\text { Not per- } \\
\text { formed }\end{array}$ & $\begin{array}{l}\text { Not per- } \\
\text { formed }\end{array}$ & $\begin{array}{l}\text { SUV of } \\
\text { RLNs }\end{array}$ & Not associated with OS \\
\hline Ohri et al. (2016) [25] & 201 & $\begin{array}{l}\text { Inoperable } \\
\text { stage IIB/III }\end{array}$ & $\begin{array}{l}\text { MTV, } \\
\text { SUVmax }\end{array}$ & $\begin{array}{l}\text { Not per- } \\
\text { formed }\end{array}$ & $\begin{array}{l}\text { Not per- } \\
\text { formed }\end{array}$ & $\begin{array}{l}\text { Median OS for patients with } \\
\text { pretreatment-MV }<93.3 \mathrm{~cm}^{3} \\
22.6 \text { vs. } 20 \text { months }(p<0.001)\end{array}$ \\
\hline Bazan et al. (2017) [13] & 230 & $\begin{array}{l}\text { Inoperable } \\
\text { stage IIB/III }\end{array}$ & MTV & $\begin{array}{l}\text { Not per- } \\
\text { formed }\end{array}$ & $\begin{array}{l}\text { Not per- } \\
\text { formed }\end{array}$ & $\begin{array}{l}\text { Median OS for patients with } \\
\text { pretreatment MTV }>32 \mathrm{~cm}^{3} \\
14.8 \text { vs. } 29.7 \text { months } \\
(p<0.001)\end{array}$ \\
\hline Ohri et al. (2017) [26] & 89 & $\begin{array}{l}\text { Inoperable } \\
\text { stage III }\end{array}$ & MTV & $\begin{array}{l}\text { Not per- } \\
\text { formed }\end{array}$ & MTV & $\begin{array}{l}\text { A } 30 \text {-month cumulative inci- } \\
\text { dence rate of } 23 \% \text { for lesions } \\
\text { above } 25 \mathrm{~cm}^{3} \text { compared with } \\
4 \% \text { for lesions below } 25 \mathrm{~cm}^{3} \\
(p=0.008)\end{array}$ \\
\hline Current study & 65 & $\begin{array}{l}\text { Inoperable } \\
\text { stage III }\end{array}$ & PT-MV & PT-MV & PT-MV & $\begin{array}{l}\text { Median OS for patients with } \\
\text { pre-treatment PT-MV }<63 \mathrm{~cm}^{3} \\
26 \text { vs. } 11 \text { months }(p<0.001), \\
\text { median OS for patients } \\
\text { with post-treatment } \\
\text { PTV-MV }<25 \mathrm{~cm}^{3} 23 \text { vs. } \\
9 \text { months }(p<0.001)\end{array}$ \\
\hline
\end{tabular}

$C T$ computed tomography, PET positron emission tomography, $S U V$ standardized uptake value, $T L G$ total lesion glycolysis, $M V$ metabolic volume, $M T V$ metabolic tumor volume, $R L N s$ regional lymph nodes 